\title{
Aufklärung und Revolutionsbegeisterung. Die katholischen Universitäten in Mainz, Heidelberg und Würzburg im Zeitalter der Französischen Revolution (1789-1792 / 1793-1803)
}

\section{Marita Gilli}

\section{OpenEdition}

\section{Journals}

Édition électronique

URL : https://journals.openedition.org/ahrf/2283

DOI : 10.4000/ahrf.2283

ISSN : 1952-403X

Éditeur :

Armand Colin, Société des études robespierristes

Édition imprimée

Date de publication : 1 juin 2002

Pagination : 248-249

ISSN : 0003-4436

Référence électronique

Marita Gilli, «Aufklärung und Revolutionsbegeisterung. Die katholischen Universitäten in Mainz, Heidelberg und Würzburg im Zeitalter der Französischen Revolution (1789-1792 / 1793-1803) », Annales historiques de la Révolution française [En ligne], 328 | avril-juin 2002, mis en ligne le 15 mai 2006, consulté le 24 avril 2022. URL : http://journals.openedition.org/ahrf/2283 ; DOI : https://doi.org/ 10.4000/ahrf.2283

Ce document a été généré automatiquement le 24 avril 2022.

Tous droits réservés 


\section{Aufklärung und Revolutionsbegeisterung. Die katholischen Universitäten in Mainz, Heidelberg und Würzburg im Zeitalter der Französischen Revolution (1789-1792 / 1793-1803)}

\section{Marita Gilli}

\section{RÉFÉRENCE}

Jorg Schweigard, Aufklärung und Revolutionsbegeisterung. Die katholischen Universitäten in Mainz, Heidelberg und Würzburg im Zeitalter der Französischen Revolution (1789-1792 / 1793-1803), Schriftenreihe der Internationalen Forschungsstelle « Demokratische Bewegungen in Mitteleuropa 1770-1850», hg. v. Helmut Reinalter, Bd. 29, Peter Lang, Frankfurt / Main, 2000, 559 p.

1 Ce volume est l'édition d'une thèse soutenue à Stuttgart sous la direction du professeur Axel Kuhn. L'objet de la recherche est la politisation des professeurs et des étudiants des trois universités catholiques de Mayence, Heidelberg et Wurzbourg. L'auteur veut montrer que, si la Révolution française a joué un rôle déterminant, la situation spécifique de ces universités a également été importante. C'est pourquoi il étudie aussi les conditions particulières à chacune de ces universités. S'il a choisi ces trois universités, c'est parce qu'elles se trouvent de part et d'autre du Rhin, que deux d'entre elles passaient pour éclairées (Mayence et Wurzbourg) et que les conditions dans lesquelles elles se trouvaient étaient assez différentes pour qu'il puisse tirer des conclusions générales. Les sources utilisées permettent une présentation originale de la 
situation. En effet, à côté des journaux et récits de voyage, il a consulté les archives des trois universités, les correspondances souvent encore privées des professeurs et des étudiants et surtout les journaux de bord des étudiants qui n'avaient encore jamais été regardés de près. Il suit sensiblement le même plan pour chaque université : étude de l'État dans laquelle elle se trouve, les réformes rendues possibles par l'abolition de l'ordre des Jésuites et leurs limites, l'impact de la Révolution française, l'attitude des professeurs, puis celle des étudiants.

2 À Mayence, le prince-électeur Friedrich Karl von Erthal avait fait venir des célébrités luthériennes pour réformer l'université dans le sens des Lumières. Avant le début de la Révolution française, elles sont déjà en conflit avec les autorités. Ce qui va les politiser définitivement, ce n'est pas seulement la Révolution française, mais la politique de lutte contre les Lumières du prince-électeur, son engagement contre-révolutionnaire (on ne lui pardonne pas d'avoir brisé la neutralité) et son accueil des émigrés. Les autorités réagissent à cette politisation par une censure et une surveillance renforcées qui amènent les intéressés à ne plus croire aux réformes et à mettre tous leurs espoirs dans la Révolution. Chez les étudiants, ce n'est qu'après 1789 qu'ils deviennent critiques. Leur politisation se renforce à partir de 1791 et devient plus ouverte après l'occupation du pays dans la mesure où ils ne risquent plus rien. Ils vont alors porter la cocarde tricolore et, pour ceux qui le peuvent, entrer au club (en effet, ils n'avaient souvent pas l'âge requis). La proportion des professeurs et des étudiants par rapport à ceux qui s'engagent dans le mouvement révolutionnaire est plus importante que celle des autres couches de la population. L'auteur pense à juste titre que cette activité eût été moindre s'il n'y avait pas déjà eu à Mayence un climat prorévolutionnaire avant l'arrivée des troupes du général Custine.

3 La situation à Heidelberg présente des différences et des similitudes. Si, entre 1789 et 1792, plusieurs professeurs manifestent leur sympathie pour la Révolution, ils ne le font pas ouvertement comme à Mayence. Là aussi, le heurt avec l'orthodoxie renforce la politisation, mais plus nombreux sont ceux qui émigrent en Alsace, éprouvant le besoin de vivre dans un pays plus libre politiquement. Certains d'entre eux ont tenté d'avoir à partir de cette ville une influence sur la situation politique en Allemagne. Contrairement à ce qui s'est passé à Mayence, ce ne sont pas les professeurs qui ont été à la tête de la politisation, car on constate dans tout le Palatinat une grande politisation de larges couches de la population. Quant à la politisation des étudiants, elle n'intervient qu'après 1792-1793, elle est moins importante qu'à Mayence, mais dure jusqu'à la fin du siècle. Elle s'accompagne d'une indifférence religieuse qui a pour conséquence une perte d'autorité du souverain "de droit divin ». Comme à Mayence, un facteur qui joue en faveur de leur politisation est la proximité de la France et la propagande qui vient de Strasbourg et ne laisse pas indifférent. La présence fréquente des troupes autrichiennes a aussi contribué à leur politisation, entraînant des positions pour ou contre. Ceux qui sympathisent avec la Révolution n'hésitent pas à le manifester ouvertement par le port de la cocarde, en s'habillant comme les sans-culottes ou en chantant la Marseillaise.

4 À Wurzbourg, les professeurs n'avaient pas pu se politiser avant la Révolution, car il n'y avait pas de sociétés (loges franc-maçonnes, société de lecture...) où ils auraient pu échanger leurs opinions. Il n'y a pas non plus de lutte contre les Lumières qui aurait pu les politiser. L'éloignement de la France fait que la propagande ne parvient pas jusquelà et que, jusqu'en 1796, le pays n'est pas concerné par la guerre. Les professeurs ne 
pouvaient donc parler politique qu'indirectement dans leurs cours, et ils n'ont pas cherché à influencer les étudiants. Chez les étudiants en revanche, il y a dès le début des années 1790 des réactions antireligieuses et libertaires. Elles se manifestent comme ailleurs par le port de la cocarde et d'habits de sans-culottes. L'afflux d'étudiants étrangers venus de la rive gauche du Rhin en 1793 permet la fondation d'un club des " amis de la liberté et de l'égalité » qui répand des tracts contre la noblesse et le haut clergé. Le "Menschheitsbund » fondé en 1794 est encore plus actif, répand des écrits politiques et cherche à mettre en place une organisation dépassant la région en s'unissant à des étudiants d'autres universités dans le but de libérer Mayence à l'aide des troupes françaises. La répression frappe durement ce groupe, ce qui fait que lors de l'occupation de la ville en 1796, ils n'ont pas d'activité politique comme à Mayence. Ce n'est qu'à partir de 1797 qu'ils manifestent de nouveau leur sympathie. L'exemple de Wurzbourg est particulièrement intéressant parce qu'il témoigne d'une forte politisation des étudiants dans un contexte difficile et leur volonté de dépasser le cadre régional.

5 L'auteur conclut qu'il y a donc eu une politisation certaine des étudiants et des professeurs dans ces trois universités, malgré leurs différences et les nuances qui les caractérisent. Le contexte joue un rôle plus important pour les professeurs; souvent, c'est la lutte contre les Lumières et leurs difficultés avec l'orthodoxie qui ont entraîné une critique politique et le regard vers la France où ils voyaient se réaliser les idéaux des Lumières. À Mayence et à Heidelberg où les professeurs étaient déjà organisés dans des sociétés, la Révolution française a été saluée avec enthousiasme, à la différence qu’à Mayence les circonstances ont permis une activité révolutionnaire alors qu'à Heidelberg, les professeurs qui voulaient être politiquement actifs ont dû émigrer à Strasbourg. À Wurzbourg en revanche, les conditions ont eu pour conséquence qu'il n'y a pas eu de sympathisants pour la Révolution parmi eux. À très peu d'exceptions près, les professeurs révolutionnaires sont en effet d'anciens Aufklärer, ce qui conforte la thèse encore parfois contestée que les Lumières ont préparé le terrain pour la Révolution. Il y a manifestement plus d'unité dans la politisation des étudiants. Leurs journaux montrent bien leur processus de prise de conscience politique. Dans les trois universités, ils n'ont pas hésité à manifester ouvertement leur sympathie. Une constante importante, surtout pour l'Allemagne, est l'indifférentisme religieux qui s'instaure et prend valeur politique dans ce pays. L'exemple de Wurzbourg montre que la politisation des étudiants est indépendante de celle des professeurs. Chez les étudiants, les sources ne mentionnent aucune attitude contre-révolutionnaire. Malgré son échec, la République de Mayence a gardé valeur de symbole pour les étudiants des autres universités puisqu'ils ont même espéré reconquérir Mayence par les armes. Il est manifeste qu'ils veulent devenir politiquement actifs. L'histoire de la politisation dans ces trois universités montre donc bien qu'en Allemagne aussi on a voulu passer de la théorie à la pratique et que les Lumières ont mené à la Révolution.

6 Si les nombreuses études sur cette période de l'Allemagne avaient déjà permis d'aboutir à de telles conclusions, celle-ci a ceci d'original qu'elle analyse dans le détail le comportement des étudiants grâce à l'exploitation de leurs journaux de bord qui n'avait jamais été entreprise. Un appareil critique et une abondante bibliographie complètent cet ouvrage ; on pourra reprocher à cette dernière d'ignorer les travaux français sur l'Allemagne et la Révolution française, ce qui est malheureusement souvent le cas chez les historiens allemands. 